A 11705 62 7263

United States Department of Commerce Technology Administration

National Institute of Standards and Technology

NISTIR 5087

Empirical Modeling of

Electromagnetic Accoustic

Transducer Data

K.J. Coakley

A.V. Clark

C.S. Hehman

$Q C$

100

.056

N0.5087 



\section{Empirical Modeling of \\ Electromagnetic Accoustic \\ Transducer Data}

K.J. Coakley

A.V. Clark

C.S. Hehman

Statistical Engineering Division

Information Technology Laboratory

National Institute of Standards and Technology

Boulder, Colorado 80303-3328

August 1999

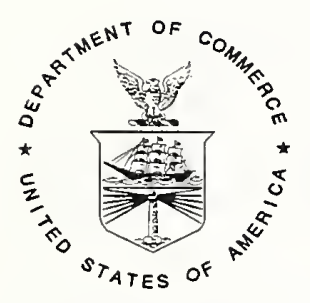

U.S. DEPARTMENT OF COMMERCE, William M. Daley, Secretary TECHNOLOGY ADMINISTRATION, Gary R. Bachula, Acting Under Secretary for Technology NATIONAL INSTITUTE OF STANDARDS AND TECHNOLOGY, Raymond G. Kammer, Director 



\title{
Empirical Modeling of Electromagnetic Acoustic Transducer Data
}

\author{
K.J. Coakley \\ Statistical Engineering Division, NIST, Boulder, CO \\ A.V. Clark and C.S. Hehman* \\ Materials Reliability Division, NIST, Boulder, CO
}

We characterize the observed phase and amplitude of acoustic birefringence data collected with an electromagnetic acoustic transducer (EMAT). Our characterization models are extended versions of an idealized model for acoustic birefringence. In the extended models, angle-dependent terms account for observed variability in phase and amplitude which is not predicted by the idealized theory. Possible sources of this extra variability include material inhomogeneity and angle-dependent sensor gain. The adjustable parameters in the model are determined by minimizing the sum of the squared phase residuals plus the sum of the squared amplitude residuals. To facilitate convergence, we determine the model parameters by fitting the extended models sequentially according to model complexity. Ten experimental data sets were collected from the same sample. We estimate the mean value of each model parameter and its associated standard error. For each extended model, we estimate the mean phase delay between the fast and slow modes. We also estimate the mean rotation angle of the pure-mode polarization directions (relative to a reference coordinate system in the specimen). From run to run, we observed phase data drift. However, the difference between the phases of the slow and fast modes did not follow this drift.

Keywords: acoustic birefringence, ultrasonics, stress determination, statistical modeling, spatial variability.

* Presently employed at MDK, Inc., Chapel Hill, NC. 


\section{Introduction}

We develop an empirical model for acoustic birefringence data collected at the National Institute of Standards and Technology (NIST). Due to acoustic birefringence, different modes can travel at different velocities within a material. We measure the interference of polarization modes which travel at different velocities within a material. Acoustic birefringence experiments yield information about the stress in a material [1-5].

In the experiments, an electromagnetic acoustic transducer (EMAT) generates an acoustic wave which is transmitted through a material. The EMAT signal is modeled as a mixture of two orthogonal polarization modes. In general, the two polarization modes travel at different velocities. According to the idealized theory, the phase and amplitude of the echo signal vary as the EMAT rotates with respect to the specimen system where the $\mathrm{x}$ (or $\mathrm{y}$ ) axis corresponds to the direction where only the fast (or slow) polarization mode is transmitted. Call the $\mathrm{x}$ and $y$ axes the pure-mode polarization directions.

In a previous study, researchers [5] showed that the idealized theory does not account for the observed variability in amplitude data. They looked at data from many echoes. They speculated that the additional variability might be due to additional scattering due to the secondary texture or layered texture in the material.

In this work, we characterize the variation of both the phase and amplitude for a particular echo as the EMAT is rotated. We quantify angle-dependent effects not predicted by the ideal model. Unlike in Reference 5, we do not analyze multiple echoes.

In our model, we allow for the phase of the slow and fast modes to depend on angle. This dependence might be due to material inhomogeneity. We also introduce terms so that the differential attenuation of the slow and fast waves depends on angle. Further, we allow for detector gain to vary with angle.

The adjustable parameters in the extended model are determined by nonlinear regression. Although material inhomogeneity and angle-dependent detector gain are plausible sources of the extra variability, there may be other physical explanations for the data. Further study is needed to resolve this question.

The paper is organized as follows. We first present the idealized theory. Second, we describe the extended model. Third, based on data collected at NIST, we model estimate model parameters and their associated standard deviations.

\section{Idealized Model}

Consider an ideal sinusoidal signal at frequency $\omega$ which is a mixture of two orthogonal polarization modes. One mode has a slow velocity of propagation, the other a fast velocity of propagation. Suppose that the signal is transmitted through the material, reflected off a boundary, and then detected. Denote the wave numbers of the fast and slow waves as $k_{f}$ 
and $k_{s}$. Call the total path length traveled $z$. We model the detected signal as

$$
s(t, z)=A \cos \left(k_{s} z-\omega t+\psi\right)+B \cos \left(k_{f} z-\omega t+\psi\right)
$$

where

$$
A=r \cos ^{2} \eta
$$

and

$$
B=r \sin ^{2} \eta,
$$

and $\psi$ is a phase angle. The angle $\eta$ is the relative angular position of the EMAT with respect to the direction where only the slow mode is transmitted in the stressed state. In general, stress causes the pure mode polarization directions to rotate by an angle $\phi$. Hence, $\eta=\theta-\phi$, where $\theta$ is the angular position of the EMAT relative to the slow direction for the unstressed state. The term $r$ is a scale factor that depends signal attenuation, signal strength, and detector gain.

In this ideal model, the slow and fast modes are attenuated at the same rate. Further, detector gain is independent of angle. The sensor is rotated as a function of time. As the EMAT rotates, $\eta$ varies. According to the ideal model, as $\eta$ varies, so do the phase and amplitude of the signal.

We express the signal $s$ as the real part of a complex signal $w$. That is,

$$
s=\operatorname{Re}(w)
$$

where

$$
w=A \exp \left(i \theta_{1}\right)+B \exp \left(i \theta_{2}\right)
$$

where

$$
\theta_{1}=\left(k_{s} z-\omega t+\psi\right)
$$

and

$$
\theta_{2}=\left(k_{f} z-\omega t+\psi\right)
$$

The amplitude and phase of $w$ are

$$
|w|=\sqrt{A^{2}+B^{2}+2 A B \cos \left(\theta_{1}-\theta_{2}\right)}
$$

and

$$
\tan (P H A S E(w))=\frac{\operatorname{Im}(w)}{R e(w)}=\frac{A \sin \theta_{1}+B \sin \theta_{2}}{A \cos \theta_{1}+B \cos \theta_{2}}
$$

Or

$\operatorname{PHASE}(w)=\tan ^{-1}(\operatorname{Im}(w), \operatorname{Re}(w))=\tan ^{-1}\left(A \sin \theta_{1}+B \sin \theta_{2}, A \cos \theta_{1}+B \cos \theta_{2}\right)$.

We estimate the amplitude and phase of the measured signal at a particular frequency by computing its Fourier transform. 


\section{Empirical Models}

We extend the idealized model by adding angle-dependent terms. We assume that the rotation rate $\left(\alpha_{2}\right)$ of the sensor is constant. The orientation of the sensor at the $i$ th time $t_{i}$ is modeled as

$$
\hat{\eta}_{i}=\hat{\alpha}_{1}+\hat{\alpha}_{2} t_{i}
$$

where $\hat{\alpha}_{2}$ is the estimated angular rotation of the EMAT and $\hat{\alpha}_{1}$ is an estimated offset angle. (In this work, if $\alpha$ is the true value of a model parameter, $\hat{\alpha}$ is the estimated value of that parameter.) To a good approximation, at the initial time $t_{1}=0$, the angular direction EMAT coincides with the slow velocity direction for the unstressed state. In the experiment, we trigger the data collection system as the rotating EMAT passes by a marked line (corresponding to the slow direction for the unstressed state). That is, at $t_{1}, \hat{\eta}_{1} \approx-\phi$ where $\phi$ is the stressed induced rotation of the slow direction. Due to timing error, we expect a slight misalignment error between the actual angular position of the EMAT at $t_{1}$ and the slow direction for the unstressed state.

The relative amplitudes (eqs $(2,3)$ ) of the slow and fast modes at time $t_{i}$ are modeled as

$$
\hat{A}_{i}=\gamma_{i} \cos ^{2} \hat{\eta}_{i}
$$

and

$$
\hat{B}_{i}=\sin ^{2} \hat{\eta}_{i}
$$

where

$$
\gamma_{i}=\left|\hat{\alpha}_{6}+\hat{\alpha}_{11} \cos \hat{\eta}_{i}+\hat{\alpha}_{12} \sin \hat{\eta}_{i}\right| .
$$

The term $\gamma_{i}$ accounts for angle-dependent differential attenuation of the slow wave relative to the fast wave. In the ideal case with no differential attenuation effects, $\gamma=1$. That is, in the ideal model, $\alpha_{6}=1$ and $\alpha_{11}=\alpha_{12}=0$.

We predict the fast and slow phases as

$$
\hat{\theta}_{1}=\hat{\alpha}_{3}+\hat{\alpha}_{7} \cos \hat{\eta}_{i}+\hat{\alpha}_{8} \sin \hat{\eta}_{i}
$$

and

$$
\hat{\theta}_{2}=\hat{\alpha}_{4}+\hat{\alpha}_{9} \cos \hat{\eta}_{i}+\hat{\alpha}_{10} \sin \hat{\eta}_{i}
$$

respectively. In the ideal case, the phases are independent of angle and $\alpha_{7}=\alpha_{8}=\alpha_{9}=$ $\alpha_{10}=0$. For the nonideal case, we allow these parameters to be nonzero. If the material is inhomogeneous, we expect the phase of the fast and slow modes to depend on rotation 
angle. In this model, $\alpha_{3}$ and $\alpha_{4}$ denote the average value of the phases of the slow and fast modes over the full 360 degrees in which the EMAT rotates.

At the $i$ th time $t_{i}$, we model the predicted phase of the signal (which is the interference of the fast and slow modes) as

$$
P H \widehat{A S E}\left(w_{i}\right)=\tan ^{-1}\left(\hat{C}_{i}, \hat{D}_{i}\right)
$$

where

$$
\hat{C}_{i}=\hat{A}_{i} \sin \hat{\theta}_{1}+\hat{B}_{i} \sin \hat{\theta}_{2}
$$

and

$$
\hat{D}_{i}=\hat{A}_{i} \cos \hat{\theta}_{1}+\hat{B}_{i} \cos \hat{\theta}_{2}
$$

The predicted amplitude of the signal (in volts) is

$$
\widehat{\left|w_{i}\right|}=\sqrt{\left|\beta_{i}\right|} \sqrt{\hat{A}_{i}^{2}+\hat{B}_{i}^{2}+2 \hat{A}_{i} \hat{B}_{i} \cos \left(\hat{\theta}_{1}-\hat{\theta}_{2}\right)}
$$

where $\beta_{i}$ is an angle-dependent scale factor

$$
\beta=\hat{\alpha}_{5}+\hat{\alpha}_{13} \cos \hat{\eta}_{i}+\hat{\alpha}_{14} \sin \hat{\eta}_{i}
$$

In the ideal case, only $\alpha_{5}$ would be nonzero. In the nonideal case, the sensitivity (gain) of the detector is allowed to vary with angular position. For a discussion of these higher order effects, see Reference 7.

\subsection{Implementation Details}

The model described in the previous section has 14 adjustable parameters. To facilitate convergence, we determine the values of the 14 adjustable parameters sequentially. Models of increasing complexity are fitted to the data sequentially. The estimated parameters determined from each model serve as initial guesses for the parameter estimates in the successive model. Model 5, the simplest model, has five adjustable parameters $\left(\alpha_{k}, k=1,5\right)$. For this model, $\alpha_{6}=1$ and $\alpha_{m}=0$ for $m>6$. In Model 10, the last 4 parameters are set to 0 but the first 10 are adjustable. In Model 12, $\alpha_{13}=\alpha_{14}=0$, but the first 12 parameters are adjustable. Finally, we fit the full model Model 14. In the simplest model (Model 5), the initial guess for the $\alpha_{5}$ is the squared value of the maximum amplitude computed from the observed data. The initial guess for $\alpha_{3}$ and $\alpha_{4}$ are the minimum and maximum phases computed from the data (Table 1). 
Table 1. Parameter bounds and initial guesses.

\begin{tabular}{cccc}
\hline Parameter & Lower bound & Upper bound & Initial guess \\
\hline \hline 1 & 0 & $\pi$ & $\pi / 2$ \\
2 & $0.09 \mathrm{~s}^{-1}$ & $0.12 \mathrm{~s}^{-1}$ & $0.105 \mathrm{~s}^{-1}$ \\
3 & -4 & 4 & $\min (\operatorname{phase}(w))$ \\
4 & -4 & 4 & $\max (\operatorname{phase}(w))$ \\
5 & $0.01 \times \max \left(|w|^{2}\right)$ & $100 \times \max \left(|w|^{2}\right)$ & $\max \left(w^{2}\right)$ \\
6 & 0.0001 & 1000 & 1 \\
7 & -0.5 & 0.5 & 0 \\
8 & -0.5 & 0.5 & 0 \\
9 & -0.5 & 0.5 & 0 \\
10 & -0.5 & 0.5 & 0 \\
11 & -0.5 & 0.5 & 0 \\
12 & -0.5 & 0.5 & 0 \\
13 & $-2.0 \times \max \left(|w|^{2}\right)$ & $2.0 \times \max \left(|w|^{2}\right)$ & 0 \\
14 & $-2.0 \times \max \left(|w|^{2}\right)$ & $2.0 \times \max \left(|w|^{2}\right)$ & 0 \\
\hline \hline
\end{tabular}

The adjustable parameters are determined by minimizing the sum of the squared phase residuals plus the the sum of the squared amplitude residuals. That is, we minimize

$$
L=L_{\theta}+L_{A}
$$

where

$$
L_{\theta}=\frac{1}{N} \sum_{i=1}^{N}\left(P H \widehat{A S E}\left(w_{i}\right)-P H A S E\left(w_{i}\right)\right)^{2}
$$

and

$$
L_{A}=\frac{1}{N V_{o}^{2}} \sum_{i=1}^{N}\left(\widehat{\mid w_{i}}|-| w_{i} \mid\right)^{2} .
$$

Above, $N$ is the number of data points and $V_{o}=1$ volt.

Before fitting the model to the data, we compute the mean phase of the measured signal over all times (or angular positions). This mean is subtracted from all phases. After we estimate the phase and amplitude of the signal as a function of angle, we add the mean phase back to the estimated phase. In the optimization, $\hat{\theta}_{1} \leq \hat{\theta}_{2}$. The program does not identify whether $\hat{\theta}_{1}$ corresponds to either the slow or the fast mode.

During fitting, the parameters are constrained to fall between lower and upper bounds (see Table 1). If the bounds are $L_{i}$ and $U_{i}$, we express the constrained parameter $\alpha_{i}$ in terms of an unconstrained parameter $q_{i}$ as

$$
\alpha_{i}=L_{i}+\left(U_{i}-L_{i}\right) /\left(1+\exp \left(q_{i}\right)\right) .
$$


We minimize $L$ as a function of the unconstrained parameters $\vec{q}=\left(q_{1}, q_{2}, \ldots q_{K}\right)$ using a quasi-Newton method where the Hessian matrix and gradient are approximated numerically [6].

As a check, the model parameters were fitted to one of the ten data sets using a different optimization algorithm. The alternative optimization algorithm was similar to the one described above except that the parameters were not constrained. That is, for each parameter, the lower and upper bounds were pushed to $-\infty$ and $\infty$. For each of the 14 parameters, the fractional agreement between the estimates provided by the two algorithms was within $5 \times 10^{-6}$. Since the estimated fractional standard deviation of each parameter due to random noise and run to run variability (Table 2) is much greater than $5 \times 10^{-6}$, the difference between the two algorithms is negligible for the data studied here.

Table 2. Parameter estimates for Model 14. (The estimated standard deviation, that is the standard error, is denoted as SE.)

\begin{tabular}{ccccc}
\hline Parameter & Mean estimate & SE of estimate & SE of mean estimate & $z_{\text {stat }}$ \\
\hline \hline$\alpha_{1}^{*}$ & 0.11119 & 0.04231 & 0.01338 & \\
$\alpha_{2}$ & $0.10479 \mathrm{~s}^{-1}$ & $0.00061 \mathrm{~s}^{-1}$ & $0.00019 \mathrm{~s}^{-1}$ & \\
$\alpha_{3}$ & -1.40222 & 0.03382 & 0.01069 & \\
$\alpha_{4}$ & -0.88195 & 0.03154 & 0.00998 & \\
$\alpha_{5}$ & $7.48664 \mathrm{~V}^{2}$ & $0.16321 \mathrm{~V}^{2}$ & $0.05161 \mathrm{~V}^{2}$ & \\
$\alpha_{6}$ & 0.97565 & 0.00706 & 0.00223 & \\
$\alpha_{7}$ & -0.02226 & 0.00365 & 0.00116 & -19.25607 \\
$\alpha_{8}$ & 0.03252 & 0.00826 & 0.00261 & 12.44404 \\
$\alpha_{9}$ & -0.02207 & 0.00774 & 0.00245 & -9.01981 \\
$\alpha_{10}$ & 0.02097 & 0.00330 & 0.00104 & 20.11370 \\
$\alpha_{11}$ & -0.02377 & 0.00636 & 0.00201 & -11.82574 \\
$\alpha_{12}$ & -0.00454 & 0.00597 & 0.00189 & -2.40581 \\
$\alpha_{13}$ & $-0.27915 \mathrm{~V}^{2}$ & $0.09533 \mathrm{~V}^{2}$ & $0.03015 \mathrm{~V}^{2}$ & -9.25945 \\
$\alpha_{14}$ & $0.06408 \mathrm{~V}^{2}$ & $0.04172 \mathrm{~V}^{2}$ & $0.01319 \mathrm{~V}^{2}$ & 4.85706 \\
$\alpha_{4}-\alpha_{3}$ & 0.52027 & 0.00641 & 0.00203 & \\
\hline \hline
\end{tabular}

\section{Observed Data}

Ten experimental data sets were collected for the same sample. The initial angular position of the EMAT was varied from run to run. On the specimen, angular markers were spaced at an interval of 10 degrees. As the EMAT rotated past a particular angular marker, the signal generator was activated. In each of the 10 data sets, the number of samples varied slightly. For each data set, the number of samples is approximately 400. In Figure 1 and 2, observed and predicted amplitude and phase for the idealized model (five parameters) and empirical 
models in increasing complexity $(10,12$, and 14 parameters) are shown for one of the 10 data sets. In Figure 3 and 4, phase residuals and amplitude residuals are shown for the same data set. From each of the 10 data sets, we compute the RMS value of the phase and amplitude residuals. From these, we compute mean RMS values and associated standard errors of these mean RMS values (Table 3). In this work, mean values and standard errors of mean values are denoted using a special notation. For instance, 0.0246(11) signifies that the mean is 0.0246 and that the associated standard error of the mean is 0.0011 .

Table 3. RMS prediction errors.

\begin{tabular}{cccc}
\hline Model order & $\sqrt{L_{\theta}}$ & $\sqrt{L_{A}}$ & $\sqrt{L_{A}^{2}+L_{\theta}^{2}}$ \\
\hline \hline 5 & $0.0246(11)$ & $0.0829(11)$ & $0.0865(4)$ \\
10 & $0.0273(11)$ & $0.0670(11)$ & $0.0724(4)$ \\
12 & $0.0144(2)$ & $0.0396(5)$ & $0.0421(5)$ \\
14 & $0.0137(1)$ & $0.0378(5)$ & $0.0402(4)$ \\
\hline \hline
\end{tabular}

For the $k$ th data set, define an adjusted rotation angle (in radians) as

$$
\alpha_{1}^{*}=\alpha_{1}-\frac{(k-1) \pi}{18} .
$$

If our prior knowledge of the initial rotation angles is correct, the adjusted rotation angles should be equal. The variability of the adjusted rotation angle appears random (Figure 5, upper left).

However, the estimated values of $\alpha_{3}$ and $\alpha_{4}$ drift upward as the initial angle is increased. We attribute this trend to time drift in the electronics. For estimating birefringence, the difference $\alpha_{4}-\alpha_{3}$ is of interest. Although both $\alpha_{4}$ and $\alpha_{3}$ follow a clear trend, the difference does not (Figure 5 , bottom right).

To better demonstrate the drift, phase data from all 10 experiments are plotted in the upper part of Figure 6. In the lower part, corrected phases are plotted. The phase data from each run are corrected by subtracting $\frac{1}{2}\left(\hat{\alpha}_{4}+\hat{\alpha}_{3}\right)$ from each phase data point. On the $\mathrm{x}$-axis of Figure 6, we plot $\hat{\eta}(\bmod 360$ degrees).

The fitted values of the angular correction factors $\hat{\alpha}_{k}, k>6$ were all statistically significant. That is, the difference between the mean value of each and 0 was not explainable by random estimation error. For each parameter, we compute the ratio of the mean value of the estimate (from all ten runs) divided by the standard error of this mean value. We define this ratio to be $z_{\text {stat }}$. When the absolute value of this ratio $\left(\left|z_{\text {stat }}\right|\right)$ is large (say greater than 2) the difference between the mean estimate and zero is statistically significant.

In Table 4, we list the values and associated mean values of the adjusted rotation angle $\alpha_{1}^{*}$ and the phase delay $\alpha_{4}-\alpha_{3}$ for the various models. From the observed data, we computed the difference between the maximum and minimum phase for each data set. The mean 
difference is 0.6126 . The estimated standard deviation of the difference is 0.0068 . Some of the variability in the rotation angle is due to timing errors. The signal generator is started by a human operator (rather than by an automatic procedure) as the EMAT rotates past an angular marker. Hence, better experimental techniques may lead to a reduction in the variability of the offest angle.

\section{Summary}

The empirical models account for variation in both the phase and amplitude of the EMAT data (at a particular frequency). The empirical models are generalizations of the idealized model. In the extended models, the following depend on angle: the phase of the slow and fast modes, differential attenuation of the slow and fast waves and the EMAT detection efficiency. From multiple measurements, we estimated the mean value of the parameter estimates and associated standard errors. All the parameters in the extended models varied from 0 by a statistically significant margin. We fitted the model to both phase and amplitude data simultaneously. As the complexity of the extended model was increased, the estimates of the adjusted rotation angle and the difference in the mean phases of the two polarization modes stabilized (Table 4).

The phase data drifted from run to run. However, the difference between the estimated slow and fast model phases (which is of interest in stress studies) did not drift. Given the parameter estimates in the extended model, we adjusted the phase data from different runs for relative drift (Figure 6).

Although material inhomogeneity and angle-dependent detector gain are plausible sources of the extra variability not predicted by the ideal theory, there may be other physical explanations for the data. Further study is needed to resolve this question.

Table 4. Adjusted rotation angle $\left(\alpha_{1}^{*}\right)$ and phase delay $\left(\alpha_{4}-\alpha_{3}\right)$ statistics.

\begin{tabular}{ccc}
\hline model order & adjusted rotation angle (radians) & phase delay (radians) \\
\hline \hline 5 & $0.0732(53)$ & $0.5269(57)$ \\
10 & $0.0982(59)$ & $0.5254(53)$ \\
12 & $0.1177(45)$ & $0.5207(66)$ \\
14 & $0.1112(42)$ & $0.5203(64)$ \\
\hline \hline
\end{tabular}




\section{References}

[1] Harris, R.V. Acoustic birefringence: methods and difficulties in materials characterization. Mater. Eval. 55(10): 1150-1161; 1997.

[2] Schneider, E. Ultrasonic birefringence effect- its application for materials characterizations. Opt. Laser Eng. 22(4-5): 305-323; 1995.

[3] Tanala, E.; Bourse, M.; Fremiot, M.; Debelleval, J.F. Determination of near surface residual stresses on welded joints using ultrasonic methods, NDTE Int 28(2): 88-88; 1995.

[4] Clark, A.V. On the use of acoustic birefringence to determine components of plane stress. Ultrasonics 23: (1) 21-30; 1985.

[5] Mignogna, R.B; Delsanto, P.P; Clark, A.V. Energy distribution for SH-waves in slightly anisotropic materials. Rev. Prog. Quant. NDE 7B: 1375-1382; 1988.

[6] Dennis, J.E; Schnabel, R.B. Numerical methods for unconstrained optimization and nonlinear equations. Prentice-Hall, Englewood Cliffs, NJ; 1983.

[7] Clark, A.V.; Hehman, C.S.; Coakley, K.J. The effect of material inhomogeneity on ultrasonic stress measurements using a small-aperature EMAT. Submitted to Ultrasonics. 

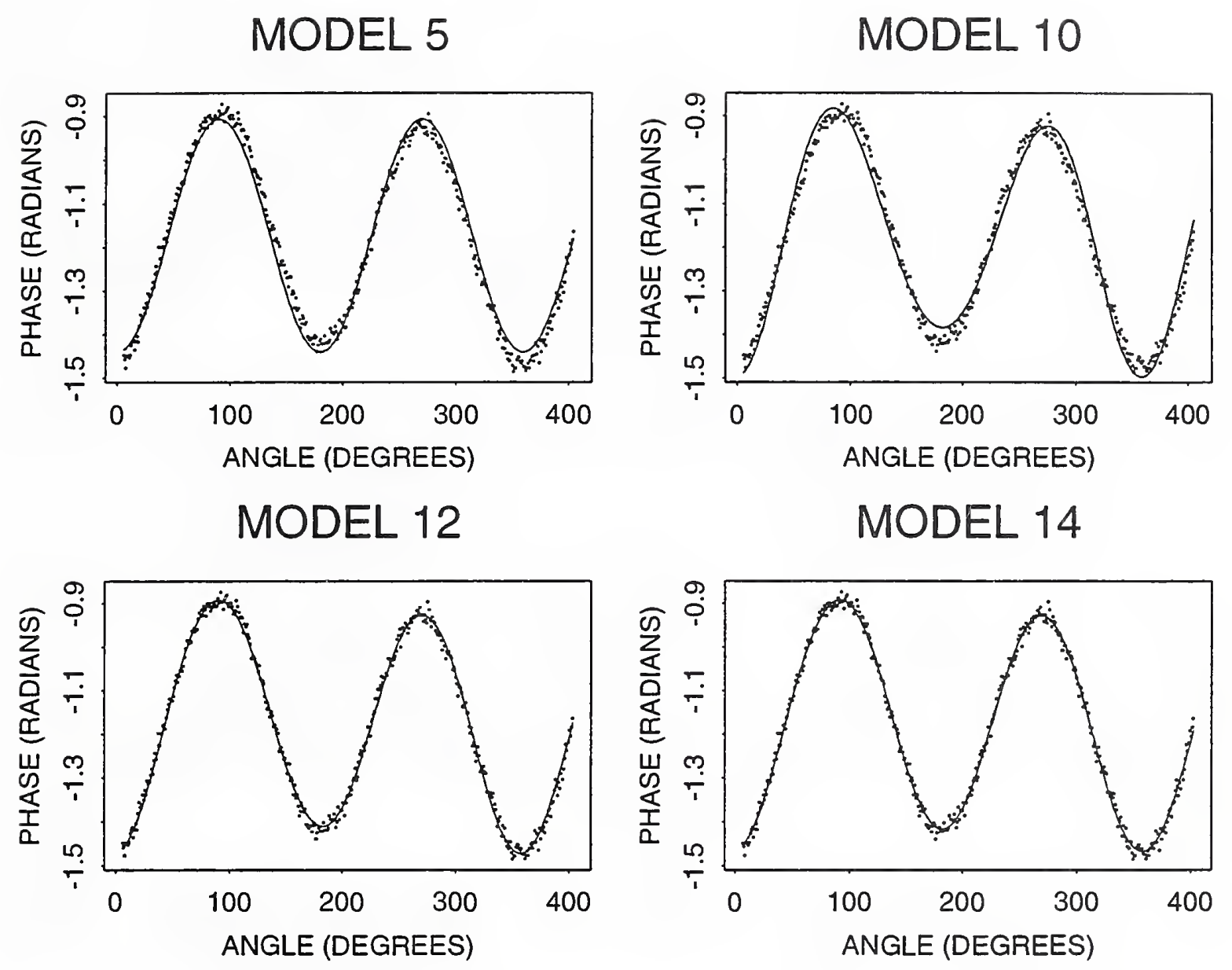

Figure 1. Observed and predicted phase for one of 10 data sets according to 5, 10, 12, and 14 parameter models. 

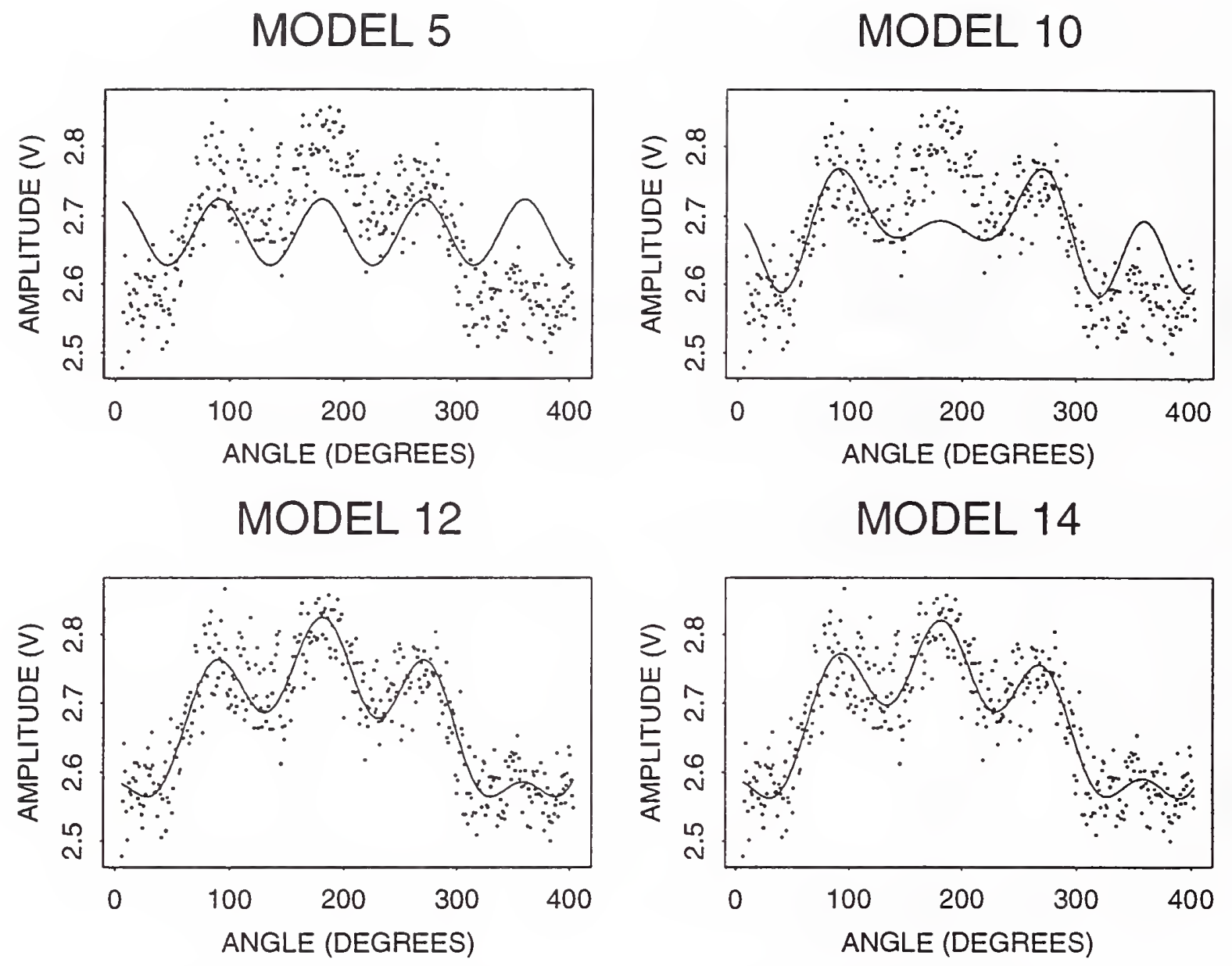

Figure 2. Observed and predicted amplitude for one of 10 data sets according to 5, 10, 12, and 14 parameter models. 

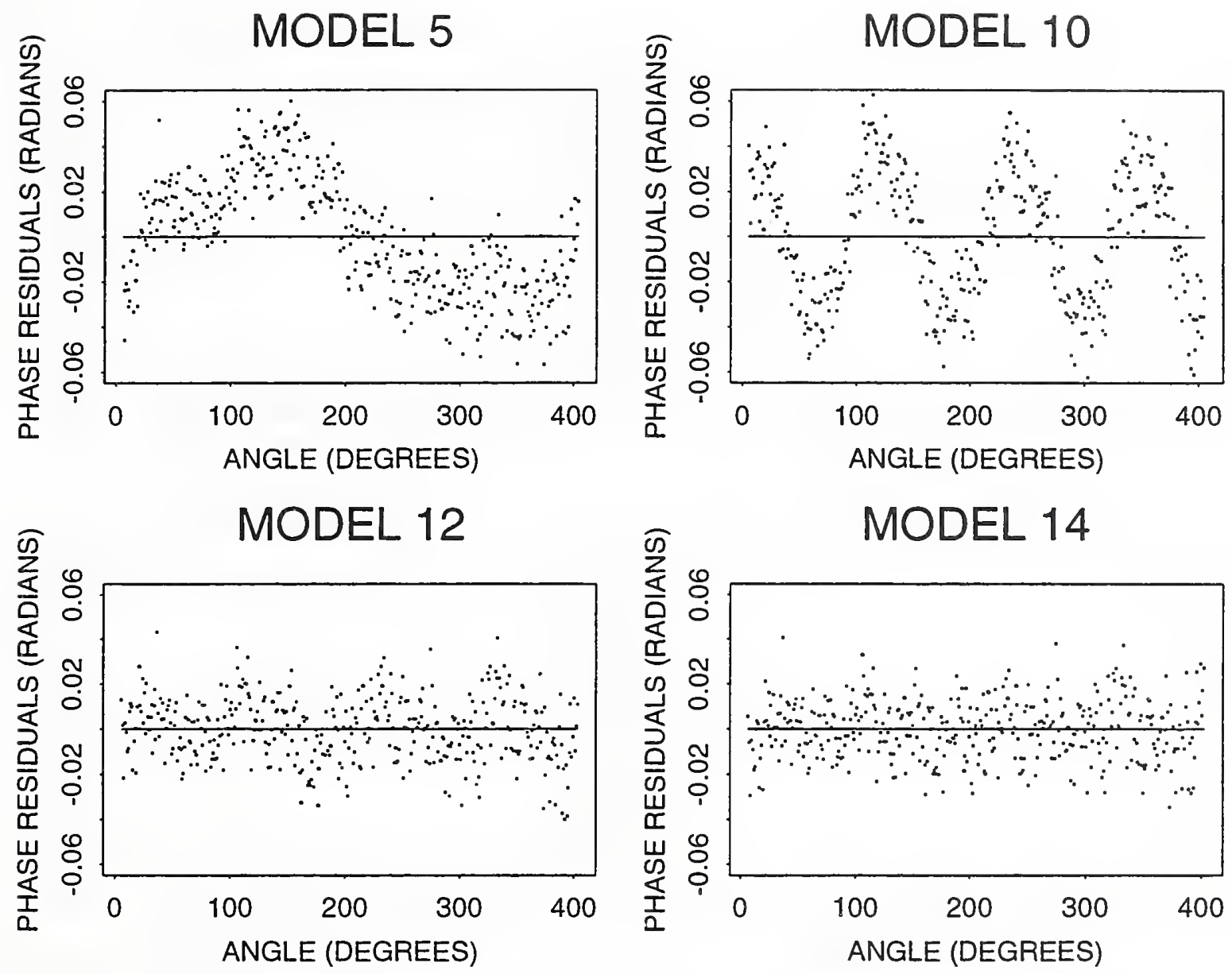

Figure 3. Phase residuals (observed-predicted) corresponding to Figure 1. 
MODEL 5

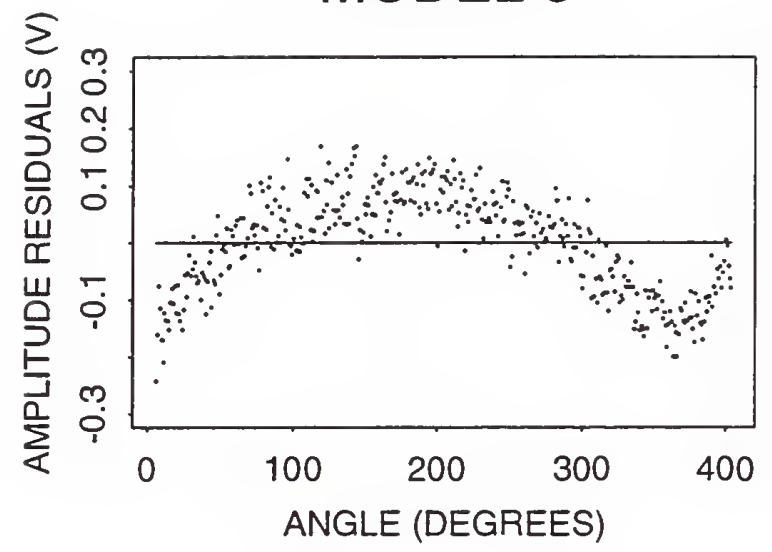

MODEL 12

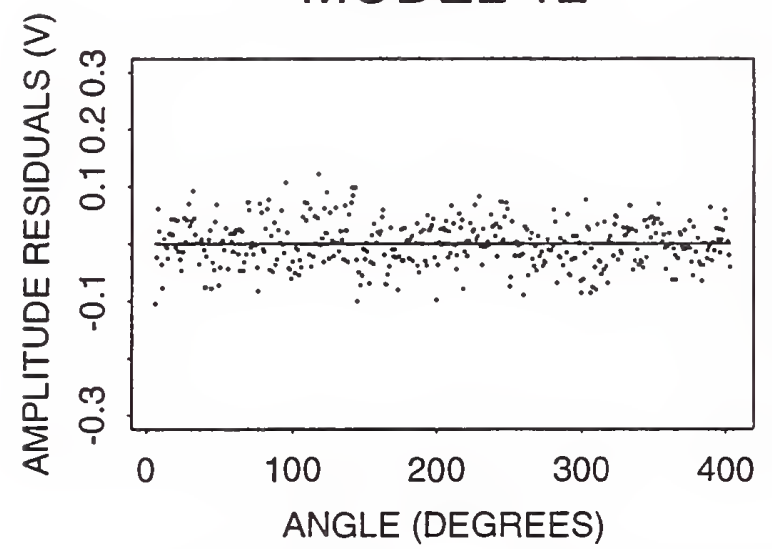

MODEL 10

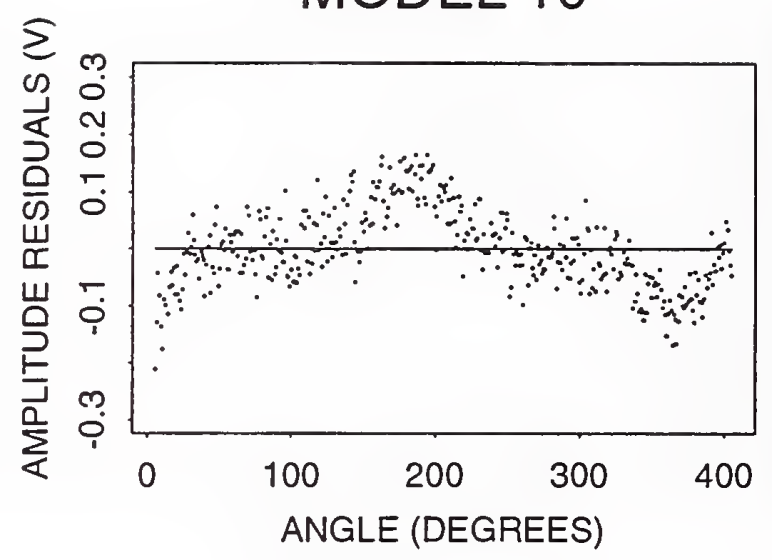

MODEL 14

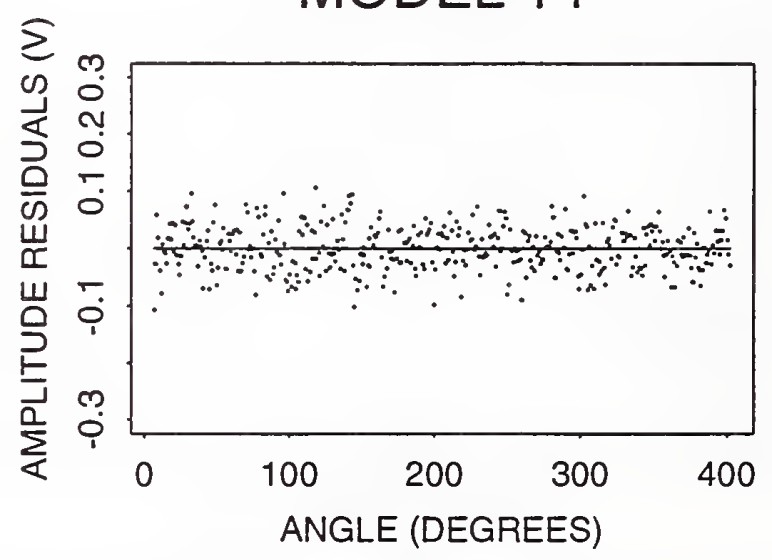

Figure 4. Amplitude residuals (observed-predicted) corresponding to Figure 2. 

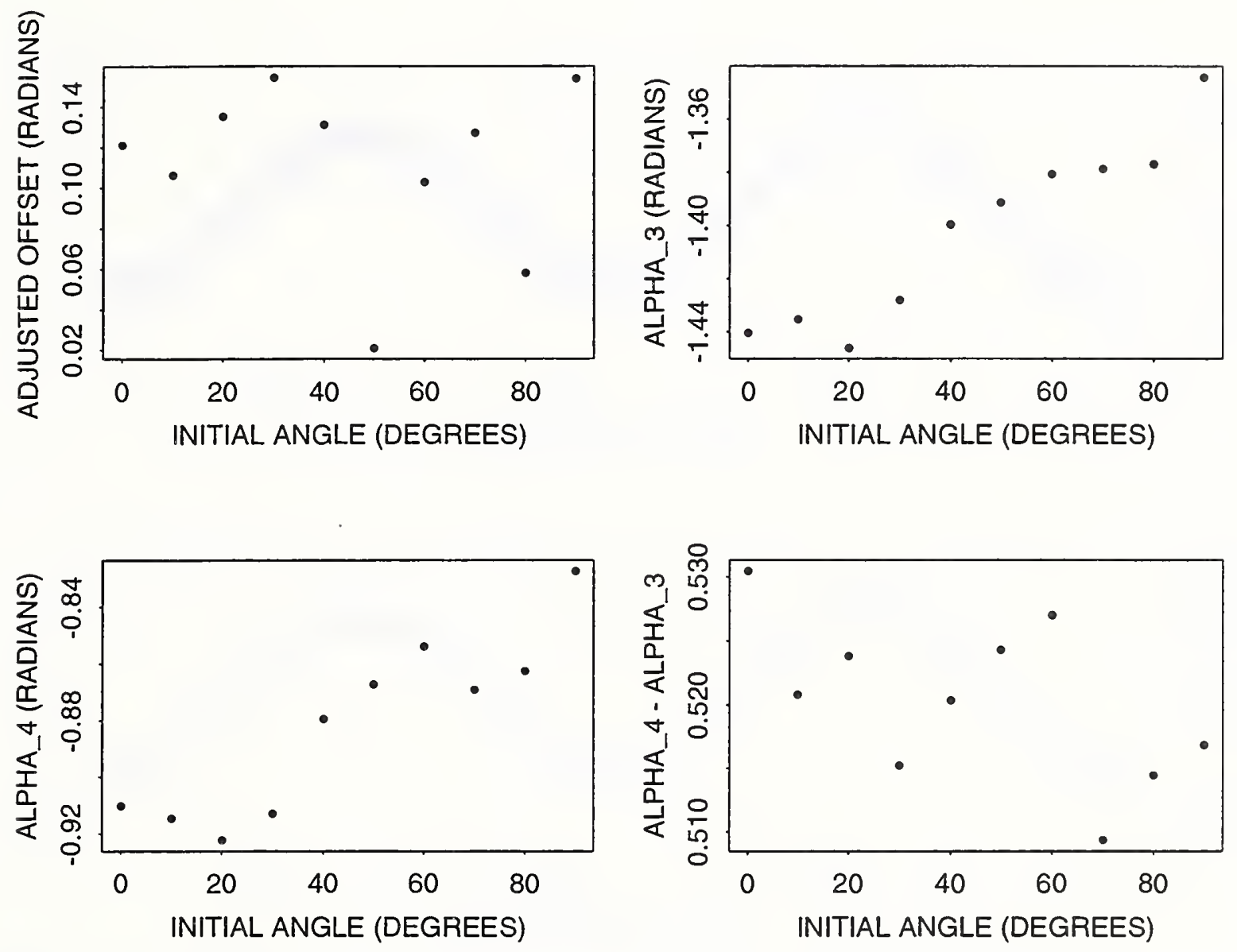

Figure 5. Upper Left: Adjusted rotation angle from each of the 10 data sets. Upper Right: Estimated value of $\alpha_{3}$. Lower left : Estimated value of $\alpha_{4}$. Lower right : Estimated value of $\alpha_{4}-\alpha_{3}$. Results obtained with 14 parameter model. 

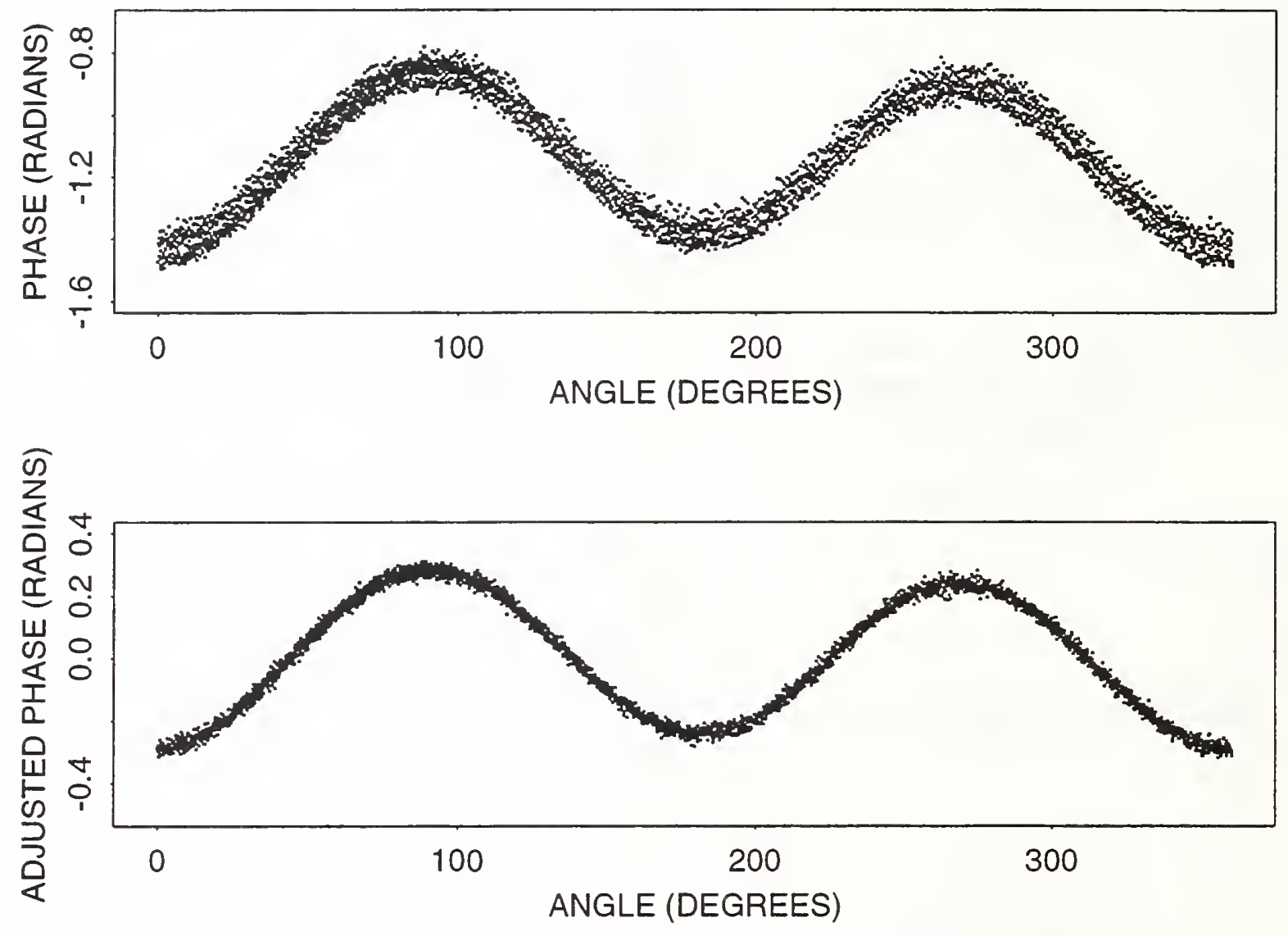

Figure 6. Top: Phase data from 10 data sets. On x-axis, we plot the angular position of the EMAT (mod 360 degrees) as predicted by 14 parameter model. Bottom: Phase data from each of the 10 data sets is adjusted to account for drift. 

